\title{
Nesting attempts and success of Arctic-breeding geese can be derived with high precision from accelerometry and GPS-tracking
}

\author{
Kees H. T. Schreven ${ }^{1^{*}}$, Christian Stolz ${ }^{2,3}$, Jesper Madsen ${ }^{4}$ and Bart A. Nolet ${ }^{1,5}$
}

\begin{abstract}
Sensors, such as accelerometers, in tracking devices allow for detailed bio-logging to understand animal behaviour, even in remote places where direct observation is difficult. To study breeding in birds remotely, one needs to understand how to recognise a breeding event from tracking data, and ideally validate this by direct observation. We tagged 49 adult female pink-footed geese (Anser brachyrhynchus) with transmitter neckbands in Finland in spring of 2018 and 2019, and in Svalbard in summer 2018, and validated inferences from tracking by field observations of nesting sites and family status in 2018-2020 (54 spring-summer tracks). We estimated nesting locations by taking the median coordinates of GPS-fixes at which the goose was motionless (overall dynamic body acceleration, ODBA $<1$ ) on days with a daily median ODBA $<1$, which approached the real nesting locations closely (within 1.6-3.7 $\mathrm{m}, n=$ 6). The start of nesting was defined as the first day on which the goose spent $>75 \%$ of time within $50 \mathrm{~m}$ of the nest, because nest site attendances steeply increased within one day to above this threshold. Nesting duration (number of consecutive days with $>75 \%$ nest site attendance) ranged between 3 and 44 days ( $n=28$ ), but was 30-34 days in confirmed successful nests $(n=9)$. The prolonged nesting of 39-44 days $(n=3)$ suggested incubation on unhatchable egg(s). Nest losses before hatching time occurred mostly in day 3-10 and 23-29 of nesting, periods with an increased frequency of nest site recesses. As alternative method, allowing for non-simultaneous GPS and accelerometer data, we show that nesting days were classified with $98.6 \%$ success by two general characteristics of breeding: low body motion (daily median ODBA) and low geographic mobility (daily SD of latitude). Median coordinates on nesting days approached real nest sites closely (within 0.8-3.6 $\mathrm{m}, n=6$ ). When considering only geographic mobility (allowing for GPS data only) nesting locations were similarly accurate, but some short nesting attempts were undetected and non-breeding tracks misclassified. We show that nesting attempts, as short as 3 days, and nesting success can be detected remotely with good precision using GPS-tracking and accelerometry. Our method may be generalised to other (precocial) bird species with similar incubation behaviour.
\end{abstract}

Keywords: Anser brachyrhynchus, ODBA, Incubation, Nesting duration, Brood, Parental care, Recess

*Correspondence: k.schreven@nioo.knaw.nl

${ }^{1}$ Department of Animal Ecology, Netherlands Institute of Ecology (NIOOKNAW), Droevendaalsesteeg 10, 6708 PB Wageningen, The Netherlands

Full list of author information is available at the end of the article

\section{Background}

The tracking of individual breeding attempts allows us to estimate population growth parameters and study individual variation in reproductive decisions which influence these parameters. In birds, the timing of breeding, especially relative to the local onset of spring, has

c) The Author(s) 2021. This article is licensed under a Creative Commons Attribution 4.0 International License, which permits use, sharing, adaptation, distribution and reproduction in any medium or format, as long as you give appropriate credit to the original author(s) and the source, provide a link to the Creative Commons licence, and indicate if changes were made. The images or other third party material in this article are included in the article's Creative Commons licence, unless indicated otherwise in a credit line to the material. If material is not included in the article's Creative Commons licence and your intended use is not permitted by statutory regulation or exceeds the permitted use, you will need to obtain permission directly from the copyright holder. To view a copy of this licence, visit http://creativeco mmons.org/licenses/by/4.0/. The Creative Commons Public Domain Dedication waiver (http://creativecommons.org/publicdomain/ zero/1.0/) applies to the data made available in this article, unless otherwise stated in a credit line to the data. 
profound effects on breeding propensity [1], clutch size and hatching success [2-4], chick growth and survival [5-7] and local recruitment [8 reviewed for geese in 9]. Generally, birds are found to time their breeding so that the peak food demand of the offspring during growth coincides with the peak in food abundance $[10,11]$ or food quality [12].

Due to climate warming, the onset of spring is advancing [13] and the highest rate of climate change on Earth occurs in the Arctic [14]. In response, Arctic-breeding migratory birds may adjust their arrival to the breeding grounds and timing of breeding to keep up with this environmental change [6], or may adjust their breeding location to a place or habitat with a more favourable phenology [15].

A remote region does not easily allow for direct observations of breeding birds. With modern tracking devices, migratory birds can be tagged in a non-breeding location and followed without direct observation to their remote breeding areas such as the Arctic [e.g., 16], while additional sensors such as accelerometers allow for detailed bio-logging to understand the birds' behaviour [e.g., 17, 18]. However, to enable the study of breeding biology in such birds, one needs to understand how to recognise a breeding event from tracking data, and ideally validate this with individuals that were observed directly, which is challenging in remote areas.

Breeding events have been detected in previous studies based on GPS data [e.g., 19, 20, 21], but additional sensors, especially an accelerometer, are expected to increase the power and precision of nest(ing) detection. Accelerometry enables us to focus on GPS-fixes, or days, on which the bird displayed an activity level or body orientation of interest [e.g., 22]. Especially for short nesting attempts, which might otherwise be indiscernible from daily roosting or foraging routines based on GPS patterns, accelerometry could increase the detection probability. Previous studies on wood stork (Mycteria americana), lesser kestrel (Falco naumanni), Mediterranean gull (Ichthyaetus melanocephalus) [21] concluded that nesting attempts shorter than 7-14 days can usually not be detected based on GPS-tracking data. Also, [19] and [20] defined nests of GPS-tracked barnacle geese (Branta leucopsis) and greater white-fronted geese (Anser albifrons) as locations from which geese stayed within $2 \mathrm{~km}$ for at least 10 days, during a defined breeding season. However, a more precise method which can detect short nesting attempts is important to distinguish a low breeding propensity from a high breeding failure. Furthermore, nesting success can be determined, by evaluating if the bird incubates the clutch long enough for it to hatch successfully.
We focus on the migratory pink-footed goose (Anser brachyrhynchus), which breeds in remote arctic regions and exhibits similar behaviour before, during and after the breeding season as many other goose species [23, 24] (see Fig. 1). During spring staging, pink-footed geese forage large parts of the day and move tens of kilometres daily between roosting and foraging sites [25]. They complete the over-sea migration from Norway and Finland to the breeding grounds in 2 days (range 1-9), arriving on the Svalbard breeding grounds mid-late May [26 and own data]. Just after arrival on the breeding grounds, they may roost for the biggest part of a day (pers. comm. J. Prop), and then forage actively on different sites before nesting $[26,27]$. We define nesting here as the combination of egg-laying, nest-building, incubation, and hatching. Pairs may visit prospective nesting sites several times before settling [28]. Once a nest site has been selected, the pair does not leave the area anymore and starts with egg-laying and nest-building immediately, with most nest-building happening up to 6 days after nest initiation [28]. The start of egg-laying occurs usually in late May to early June, but ranges from mid-May to mid-June [3, 24, $29,30]$. The clutch is completed with commonly $3-5$ eggs (range 1-8) around day 5-9 after nest initiation, when incubation sharply increases from $40-60 \%$ to $90-100 \%$ of time [3, 24, 28-30]. Incubation is solely carried out by the female, in a sedentary posture with the head forward or leaning on the back, while the male stands nearby. The incubation period until hatching is 25-28 days, during which females usually leave the nest to feed once per day $[3,24,29,31,32]$. All chicks in one nest hatch within $24 \mathrm{~h}$, and the family leaves the nest within 36-48 h, or even within 12-24 h if disturbed [28, 29 pers. obs. J. Madsen). During this period, the female may forage with the chicks around the nest, but also go back to the nest to brood the chicks [28]. After incubation, females spend much time feeding and little time vigilant [33]. Wing moult in adults starts 2-3 weeks after hatching, 1 week earlier for non-breeders and renders birds flightless for 25 days in July-August [24, 29]. During moult, geese mainly forage, preen and roost [34]. Geese depart from Svalbard to Norway around mid-September [35, own data].

We use the above descriptions of breeding behaviour in this species to define characteristics of nesting behaviour that can be measured quantitatively by GPS-tracking and accelerometry, and thereby extract nesting locations and durations for 49 adult females, tagged in Svalbard and Finland. We ground-truth this by direct observation of nesting sites on the Svalbard breeding grounds in summer and family status on subsequent autumn, wintering, and spring staging sites. We further assess how nesting failure covaries with nest attendance. We believe that our 


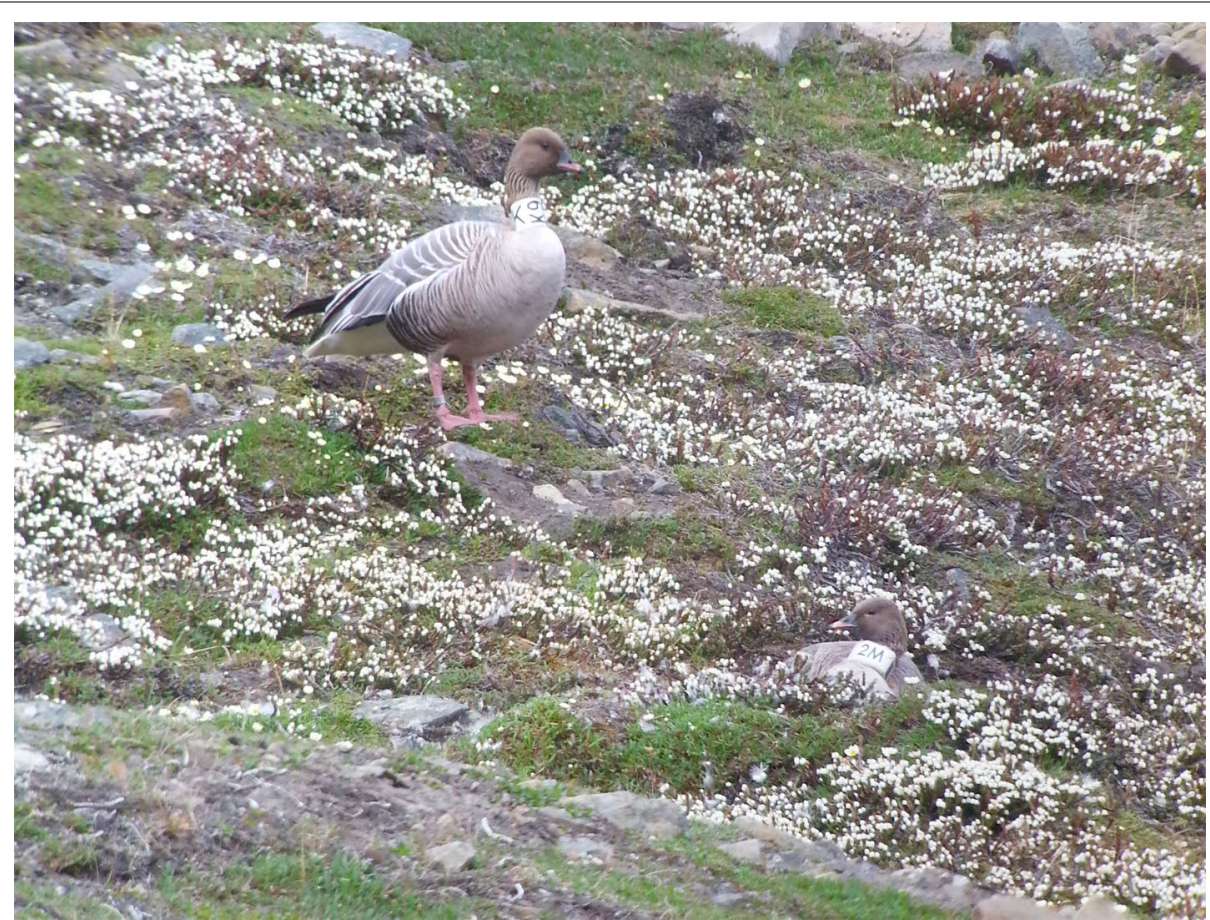

Fig. 1 A pink-footed goose with a transmitter neckband. Incubating female '2M' (solar-powered GPS-GSM transmitter neckband) and guarding male 'KK9' (normal plastic neckband) at their nest site in Endalen, Longyearbyen, Svalbard, 29 June 2020. Photo: Christian Stolz, colour-edited by Stijn Schreven

method can also be used in other (precocial) bird species with similar incubation behaviour.

\section{Methods}

\section{Catching geese}

Pink-footed geese were tagged in Svalbard and Finland. In Svalbard, geese were caught during wing moult, in family groups, on 30 July 2018 (the lake of Isdammen, Longyearbyen, $78^{\circ} 12^{\prime} 12.7^{\prime \prime} \mathrm{N}, 15^{\circ} 48^{\prime} 10.3^{\prime \prime} \mathrm{E}$ ) and 1 August 2018 (the coastal plain of Daudmannsøyra, $78^{\circ} 13^{\prime} 16.6^{\prime \prime} \mathrm{N}$, $\left.13^{\circ} 04^{\prime} 10.8^{\prime \prime} \mathrm{E}\right)$. More details on catching and handling procedure are given in [34]. The capture and tagging caused the birds to increase their amount of time spent preening, but this effect disappeared within 6-7 days after tagging [34]. In Finland, geese were caught during spring migration, by canon-net on agricultural fields in the novel staging area in Tyrnävä, Oulu $\left(64^{\circ} 49^{\prime} 33.1^{\prime \prime} \mathrm{N}\right.$ $25^{\circ} 33^{\prime} 25.1^{\prime \prime} \mathrm{E}$, see also [36]) on 28 April 2018, 27 April 2019 and 1 May 2019.

Geese were sexed by cloacal examination in the field, which was validated molecularly following [37], using blood taken from medial metatarsal vein, primer pair 2550F/2718R and the PCR-programme of [38] and running results on a $2 \%$ agarose gel.

In total, 56 geese received a transmitter in Svalbard $(n=35)$ and Finland $(n=21)$, of which 49 were females and 7 males. Here, we focus on females, although limited data on males suggest the method also works when using males. Of the 49 female geese, we obtained 54 tracks during the breeding season, over 3 years (2018: 4, 2019: 29, 2020: 21).

\section{GPS-tracking and accelerometry}

We used solar-powered GPS-GSM transmitter neckbands, type OrniTrack-N38 (Ornitela UAB, Lithuania) with a weight of $38 \mathrm{~g}$ (c. $1.5 \%$ of body mass) and an inner diameter of $38 \mathrm{~mm}$ (see Fig. 1). Tags were white and had a black two-digit individual code that could be read from a distance in the field. The tags recorded a GPS-fix every $10 \mathrm{~min}$ (when battery voltage is $75-100 \%$, 4000-4150 mV), $20 \mathrm{~min}(50-74 \%, 3850-3995 \mathrm{mV})$, $30 \mathrm{~min}(25-49 \%, 3760-3845 \mathrm{mV})$ or $60 \mathrm{~min}(<25 \%)$. In addition, when the voltage was above $85 \%(4060 \mathrm{mV})$, instead of a single GPS-fix a GPS-burst of 10 fixes was taken at $1 \mathrm{~Hz}$, to increase accuracy of elevation and speed measurements.

Immediately after each GPS-fix, or GPS-burst, a 2-s accelerometer measurement burst was taken at a frequency of $20 \mathrm{~Hz}$. Gravitational acceleration (unit g) was measured in three dimensions: along the neck (y-axis), and around the neck ( $\mathrm{x}$ and $\mathrm{z}$-axes, which are 
interchangeable due to free rotation of the neckband). Battery voltage level was recorded with each GPS-fix.

For analysis, a subset of data was taken in which, besides the single GPS-fixes, only the first GPS-fix of each GPS-burst was kept, to keep data quantity and quality homogeneous. Further subsetting was not necessary, as the variation in logging interval on the level of interest (i.e. the within-individual within-day level) was limited: on average only $7 \%$ of days contained multiple different logging intervals and on these days on average only $17 \%$ of fixes had a different logging interval than the most common logging interval length of that individual on that day. Overall, $98.3 \%$ of fixes were taken at 10 -min interval, $1.3 \%$ at $20-\mathrm{min}, 0.4 \%$ at $30-\mathrm{min}$, and $0.1 \%$ at intervals of $1 \mathrm{~h}$ or longer. Overall, voltage levels were on average $95.6 \pm$ SD 7.8\% (range 0-100\%).

The precision of a GPS-fix was evaluated with two tags placed on a fixed known coordinate, i.e. a wooden pole at Adventdalen weather station during 4-9 August 2019. This showed that $24 \%$ of GPS-fixes was within $5 \mathrm{~m}$ of the pole, $47 \%$ within $10 \mathrm{~m}, 74 \%$ within $20 \mathrm{~m}$ and $96 \%$ within $50 \mathrm{~m}$ ( $n=1424$ fixes $)$.

\section{Field observations}

For ground-truthing, nesting and roosting/foraging locations of geese on their breeding grounds were observed in Adventdalen, Svalbard, during 20 June-11 July 2020. These locations were identified with a rough procedure: selecting condensed clusters of GPS-fixes on a map, and taking the average coordinates per cluster. A cluster was defined as a collection of GPS-fixes showing that a goose frequently revisited an area with a diameter of $20-50 \mathrm{~m}$, for at least 2 days, with relatively few fixes elsewhere during this period, and occurring within the time frame of arrival (mid-late May) until the usual hatching period (late June). This procedure included all nesting sites as identified by the final procedure below, but also additional roosting/foraging sites. In the field, these sites were observed from a distance (to check presence of a tagged goose, possible partner and chicks), and visited (if no goose was present). In the majority of cases, female geese were not flushed from the nest by the observer; only in two cases, the female left the nest. From a distance it was observed that the female returned to the nest site. During a visit, the surroundings within $20 \mathrm{~m}$ of each site were checked for the presence of a nest, of which the coordinates were then taken with a handheld GPS device (GPSMAP 64 s, $\left.\operatorname{Garmin}^{\circledR}\right)$. For each nest, the number of eggs and presence of egg remains in and around the nest was noted. Egg remains were evaluated as coming from hatched nests if a thick membrane was present, yolk remains and predator bite marks were absent, and if they were trampled in the lining of the nest. Remains from depredated eggs were recognised by an irregular shape, presence of bite or peck marks, yolk remains and/or a weak thin membrane. If no eggshells were present, the nest was assumed to be depredated [3, 24, 29].

These field observations resulted in direct observations of two geese incubating on their nests, and checks of 13 sites of in total seven geese (six geese with one site each, one goose with two sites and one goose with five sites). These sites concerned six confirmed nests (one per goose, including the two nests where incubating geese were observed), five roosting/foraging areas (all of one goose), and one uncertain site, which was excluded (i.e. first site of the goose with two sites). Of the six confirmed nests, three had hatched and three were depredated. The uncertain site was attended by the goose for $>75 \%$ of time within $50 \mathrm{~m}$ on both 1-2 June 2020, whereas the goose's second site, a confirmed nest $302 \mathrm{~m}$ away, was attended for $>75 \%$ of time within $50 \mathrm{~m}$ on all 11-13 June 2020 . This uncertain site might also have concerned a nest, as the time and distance between the sites corresponds to recent findings about replacement clutches of early-failed Barnacle geese (Branta leucopsis, pers. comm. Jouke Prop, Nordenskiöldskysten, Svalbard). However, replacement clutches are not known for pink-footed geese [24, $29,30]$ but are difficult to observe, as early-failed nests have only accumulated little down [28] that can be blown by wind. At this uncertain site, only goose droppings were found.

We further increased the sample size of confirmed successful geese by seven, based on observations of geese after nesting on Svalbard and during autumn migration in Norway, Sweden, Denmark, Netherlands, and Belgium entered into the citizen science platform www.geese.org [39]. In geese, partners migrate together and with their fully fledged brood, enabling an evaluation of breeding success and pair status in autumn and even up to early spring when juveniles leave their parents [e.g., 40]. The four tracked females in 2018 were seen without chicks in autumn. Of the 29 females tracked in 2019, one was seen with chicks in summer and/or autumn, 28 without. Of the 21 females tracked in 2020, eight were seen with chicks in summer and/or autumn, and 13 without. Thus, the total sample of confirmed nesting geese was 13, of which ten were successful and three had failed.

We further increased the sample size of roosting sites by including geese roosting just upon arrival at the breeding grounds, as nesting then is physiologically not yet possible [41]. Therefore, if potential 'nesting' sites were detected (by the final procedure below) within 3 days after arrival, they were regarded as roosting sites ( $n=4$ geese). From the five roosting sites checked in the field, described above, only one was detected as potential 
'nesting' site, and therefore the total sample of roosting sites was five.

\section{Deriving nesting attempts}

Nesting attempts were derived from GPS tracking and accelerometry by identifying cut-off values of several quantifiable characteristics that follow from the breeding behaviour descriptions in the background. We selected May-July for analysis, i.e. from spring staging and migration until halfway moult and/or chick rearing, and used the tracks of confirmed nesting geese. We developed two approaches suitable for different data structures (see Fig. 2 for a schematic representation of the approaches).
For our first and main approach, simultaneously recorded GPS and ACC data is needed (Fig. 2a). As nesting is characterised by prolonged periods of sitting still, we expected to measure for breeding geese a time window of consistently low values of body motion. As proxies for body motion, overall dynamic body acceleration (ODBA) and vectorial dynamic body acceleration (VeDBA) are in use [42]. Although VeDBA is mathematically better in line with theory of acceleration [42], in our case, ODBA gave clearer contrasts for a goose within a season, and we therefore used ODBA. We first calculated the static acceleration for each of the three dimensions $(\mathrm{x}, \mathrm{y}, \mathrm{z})$ within a burst as the average raw measured (a) Simultaneous GPS and ACC data

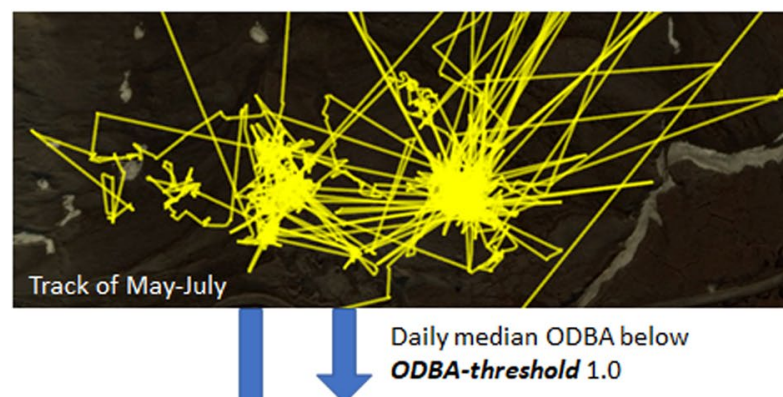

Days on which goose was mostly motionless

Individual fix $\mathrm{ODBA}<1.0$

Motionless fixes on days on which goose was mostly motionless

\begin{abstract}
Attendance
\end{abstract}
threshold:

$>75 \%$ of daily

time spent

$<50 \mathrm{~m}$ of

possible nest

\section{rose}

Median latitude and longitude

Possible nest location (b) Independent GPS and ACC data, or GPS only

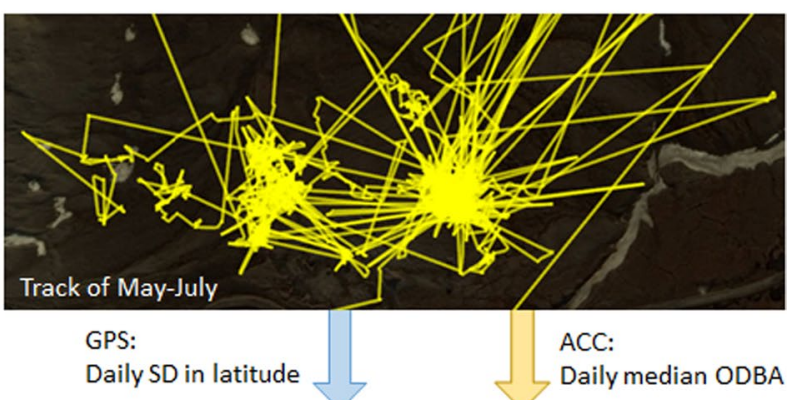

Daily geographic mobility

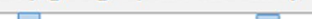

Daily body motion

$<89.4 \mathrm{~m}$

(when daily body

motion <1.482)

OR

$<15.2 \mathrm{~m}$

(when daily body

$<25.4 \mathrm{~m}$ motion $\geq 1.482$ )

Days on which goose possibly nested

Period threshold: $\geq 3$

subsequent days

Nesting duration: $30-34$ days

Successful nest

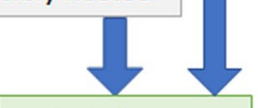

Certain nest

(1)


acceleration of each dimension within the burst. We then subtracted for each dimension $(x, y, z)$ the static acceleration from the raw measured acceleration. This gives the dynamic acceleration, of which then the absolute value was taken. The absolute values were then summed for all 40 points of all three dimensions within a 2 -s burst to get the ODBA of that burst. We defined an ODBA threshold to identify periods of 'motionlessness' based on the direct observation of geese incubating their clutch $(n=2)$ and other geese that were confirmed to have nested $(n=11$, see "Field observations" section). As geese attend their nest almost continuously during nesting, we determined the potential nesting location of each goose by taking the median values of the longitude and latitude of a subset of GPS-fixes. The subset was made by selecting the days on which the goose was predominantly motionless (i.e. daily median ODBA below threshold), and then taking from these days all GPS-fixes during which the goose was motionless (i.e. ODBA below threshold). The standard deviation of the coordinates of the selected fixes was used to evaluate the precision of the derived potential nesting site. We assessed nest site attendances by calculating the distance of each GPS-fix of a goose (also on days on which the goose was not mostly motionless) to its potential nesting location and then reporting the daily amount of time that the goose spent within a radius of $5,10,20$, and $50 \mathrm{~m}$ of its potential nest site. The radius of $50 \mathrm{~m}$ gave the clearest contrasts over time and was used in further analysis. We then defined an attendance threshold: a daily proportion of time spent within the radius of the nest that is typical of nesting. We derived this by evaluating for confirmed nests $(n=13$, see "Field observations" section) if there was a steep increase in nest site attendance, which was defined as the start of nesting, and what values characterised attendance before and after the start. Incubation may be confused with roosting behaviour (i.e. geese also sitting still, resulting in low ODBA), but roosting is expected to happen in shorter episodes and not always on the same location. Therefore, we calculated the nesting duration as the number of consecutive days on which the goose showed above-threshold attendance to its potential nesting location. In order to exclude roosting locations and identify the location as a real nest, the nesting duration should be above a minimum number of days, i.e. the period threshold. To define the period threshold, we compared roosting locations $(n=5)$ and nesting locations ( $n=13$, see "Field observations" section). If a goose spent fewer days than the period threshold at its potential nesting site, roosting and nesting could not be distinguished.

Our second and complementary approach does not require that GPS and ACC data were collected simultaneously, and can use GPS only as well. We evaluated how well nesting can be predicted by two general characteristics that geese display during nesting: low geographic mobility and low body motion, summarised on a daily level (see Fig. $2 b$ for a schematic representation). Geographic mobility was measured by the daily standard deviation of latitude of GPS-fixes. Longitude was not analysed, as the daily SD in latitude and daily SD in longitude were strongly correlated $(r=0.92, t=165.03$, $d f=4797, p<0.0001)$. We compared daily median ODBA and daily SD of latitude between different stages of the annual cycle, for each goose based on its GPS and accelerometer data: spring migration (from May 1st until reaching the breeding grounds), pre-nesting (defined as the period between spring migration and nesting), nesting (as determined by our first approach above), post-nesting (after nesting until the end of July), and for non-breeding geese the summering period as one stage (i.e. from spring migration until the end of July). Stages were compared using Linear mixed models (LMM) using the R-package "lme4" [43] on natural logarithm-transformed data to approach normality. A random effect of track ID (i.e. each year separately for each individual, $n=$ 54) was included and p-values and degrees of freedom were estimated by Satterthwaite's method (R-package "lmerTest") [44]. We then established a procedure to classify days as either 'nesting' or 'non-nesting' using Recursive Partitioning and Regression Trees (R-package 'rpart') [45]. We split our data randomly into a training set (50\%), on which the tree was based, and a testing set (50\%), with which the tree was validated. We tested two different classification trees: (1) using only geographic mobility as a single predictor, or (2) using both geographic mobility and body motion as predictors. The comparison of the two classifications allowed us to assess the added value of sensor data as opposed to GPS data without accelerometry. Following both classifications, nesting locations were determined by taking the median coordinates of nesting days, and compared ground-truthed nest coordinates $(n=6)$. After determining possible nesting sites with this second approach, real nests could be identified by again using the attendance threshold and period threshold as explained in the first approach. All statistical tests were performed in $\mathrm{R}$ [46].

\section{Deriving nesting success}

To derive nesting success-binary, i.e. whether any chicks hatched from a clutch or not-we defined a range in nesting duration typical of successful nests (Fig. 2a). This was achieved by comparing confirmed successful geese $(n=$ 9) with confirmed unsuccessful geese $(n=3)$ and breeding geese with unknown success, i.e. geese that were breeders according to our first approach, but not seen with young in autumn ( $n=16$, see "Field observations" 
section). One successful goose was excluded here because of tag failure from halfway incubation onwards. We expected the nesting duration of successful geese to be 30-38 days, i.e. 5-9 days egg-laying, plus 25-28 days incubation, plus 0-1 day hatching (see "Background" section). Any nesting duration outside this range would imply complete hatching failure.

To assess whether any nest with unknown nesting success, but a nesting duration typical of successful nests could be assumed to be successful, we analysed the geographic mobility and body motion during the post-nesting stage. We compared successful breeders, i.e. with chicks throughout summer $(n=9)$, breeders that failed during nesting, i.e. without chicks in summer $(n=16)$ and geese with a nesting duration typical of hatched nests, but with unknown hatching success and without chicks by the time of autumn migration $(n=3)$. Additionally, one goose nested successfully but lost its chicks during summer or autumn. We used LMMs with a random effect of track ID, on natural logarithm-transformed data.

\section{Variation in nest site attendance}

As we expected to find a range of nesting durations (relating to nests that had been depredated, hatched, or deserted), we aimed to better understand the moment in the nesting cycle at which nests failed. For this, we evaluated variations in the daily number and duration of nest site recesses, i.e. bouts of time that a goose spent beyond $50 \mathrm{~m}$ away from its nest. We divided the nesting cycle before possible hatching into three periods: day $0-10$, day $11-19$, and day $20-29$ after nest initiation. For each goose, we selected the period from its first nest site visit to the day before the end of nesting, and calculated per day the number and average duration of bouts spent beyond $50 \mathrm{~m}$ away from the nest. When a bout spanned 2 days, we included the bout only on the day on which it started. Bout duration was calculated by taking the time difference between the first and last GPS-fix within the bout, plus $10 \mathrm{~min}$. Thereby, bouts with only one GPS-fix were assumed to have lasted $10 \mathrm{~min}$, as the GPS-logging interval during nesting was nearly always $10 \mathrm{~min}$ (median voltage during pre-nesting and nesting stages $99.0 \%$, mean $96.4 \pm$ SD 6.7\%, range 33-100). Both the number and duration of recesses of all nesting geese were then compared between the three periods in the nesting cycle, using LMMs including a random effect of track ID.

\section{Results}

\section{Extracting nesting locations and nest attendance}

The ODBA threshold was defined at 1.0, because the daily median ODBA was below this value on $75 \%$ of days during prolonged time windows of motionlessness, typical for nesting (283 out of 377 days, $n=13$ nesting geese, Figs. 3a, c; 4a), During such time windows, geographic mobility was consistently low as well (Figs. 3b; $4 a)$. Our method to extract nesting locations reproduced the field-recorded nesting locations well, i.e. on average within $2.7 \mathrm{~m}$ (SD $=0.9$, range $1.6-3.7 \mathrm{~m}, n=6$ nests). Per goose, the SD of latitude in the subset of GPS-fixes from which the nesting location was derived was on average $9.7 \pm$ SD $4.9 \mathrm{~m}$ (range 5.6-20 m). The subset of one specific goose with a prolonged incubation period showed an SD for latitude of $635 \mathrm{~m}$, but the real nest site was still approximated within $1.7 \mathrm{~m}$. For all identified roosting episodes ( $n=5$ geese), the SD of the subset of GPS-fixes from which the roosting location was derived was on average for latitude $90.1 \pm$ SD $153 \mathrm{~km}$ (range $54.5 \mathrm{~m}-356 \mathrm{~km}$ ). The large values were generated by geese that were motionless for most of the day because of roosting, but moved between different roost sites, of which some were several hundred kilometres apart when the goose had just arrived in Svalbard.

For confirmed nesting geese (13 nests), the time a goose spent within $50 \mathrm{~m}$ from the nest steeply increased within one day by on average $70 \pm$ SD 17\% (range 35-93\%), namely from a site attendance of on average $23 \pm$ SD $21 \%$ (range $0-65 \%$ ) to on average $94 \pm 7 \%$ (range $81-100 \%$, Figs. 3d; 5a). Therefore, the attendance threshold was set at $75 \%$ of the time spent within the $50 \mathrm{~m}$ radius of the site. The period threshold was set at 3 days, as confirmed nesting geese showed nesting durations of 3-44 days, i.e. subsequent days with $>75 \%$ nest site attendance ( $n=12$ nests), whereas roosting geese showed maximally 1 day meeting the attendance threshold ( $n=5$ geese).

\section{General characteristics during breeding: body motion and geographic mobility}

During May-July, body motion (daily median ODBA, log-transformed) of female geese plotted against their geographic mobility (daily SD in latitude, log-transformed) showed clear clusters that corresponded to different stages of the annual cycle (Fig. 4). The nesting stage was characterised by lower daily median ODBA than all the other stages combined (Table 1, LMM, $t=$ 86.77, $d f=4597, p<0.0001)$. Also, the geographic mobility was lower during nesting than all other stages combined (Table 1, LMM, $t=52.09, d f=3961, p<$ $0.0001)$. Classification success into nesting versus nonnesting days was $96.4 \%$ when using only geographic mobility as predictor, and $98.6 \%$ when using both geographic mobility and body motion as predictors (Fig. 4). From the 29 breeders determined by the main approach, 26 were recognised as such by GPS-only (i.e. showing at 

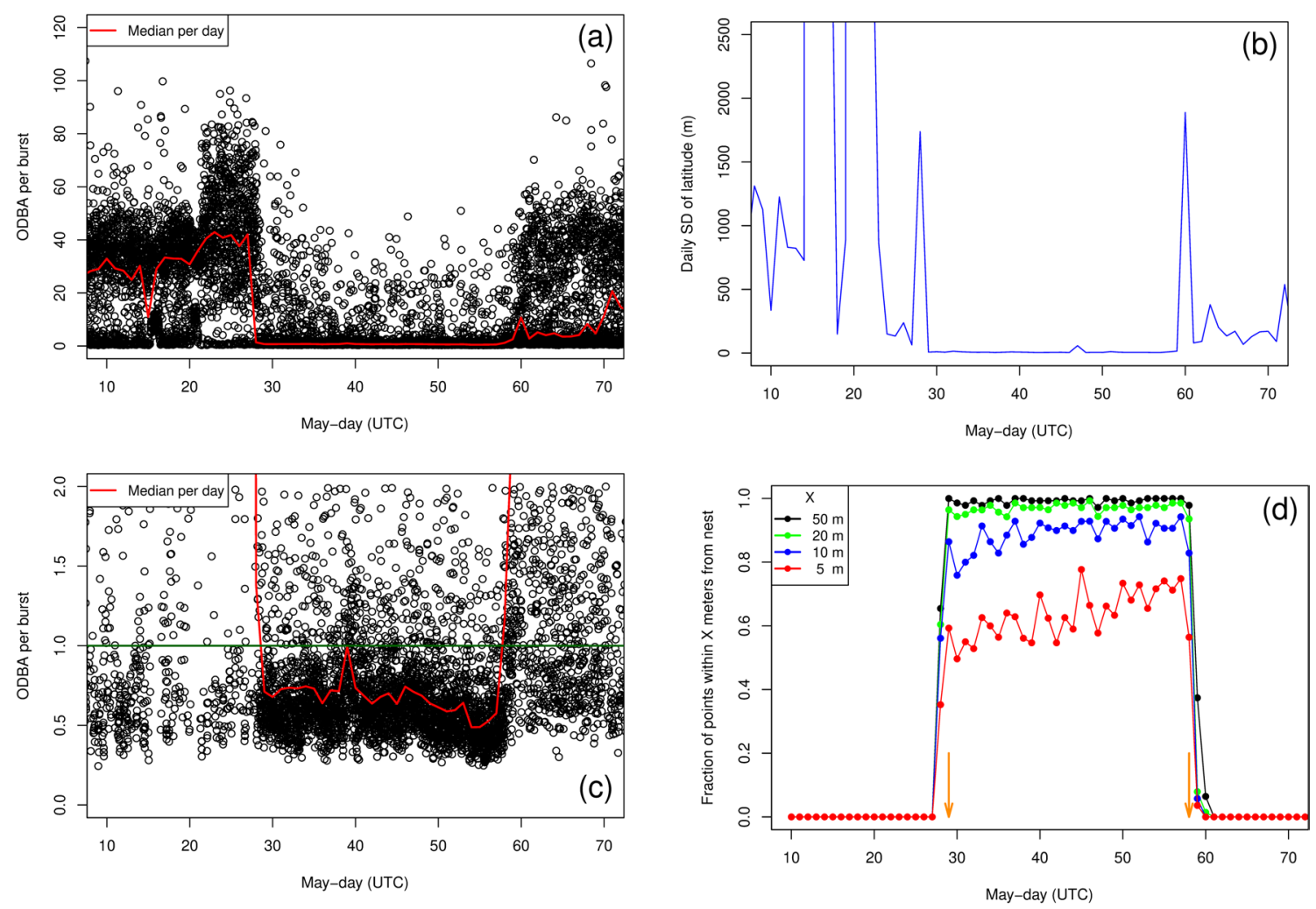

Fig. 3 Body motion, geographic mobility, and nest attendance of a pink-footed goose during spring and summer. Overall dynamic body acceleration (ODBA) per burst varied largely but showed during nesting a time window of motionlessness (a, c: zoomed in), during which geographic mobility was also notably low (b). GPS-fixes with an ODBA $<1$ on days with median ODBA $<1$ were selected to extract the nesting site by taking median coordinates (c), from which then nest site attendance was calculated (d). Inferred start and end of nesting are indicated by orange arrows in d, given by a threshold of daily attendance above $75 \%$ within $50 \mathrm{~m}$ from the nest. This nest was confirmed to have hatched
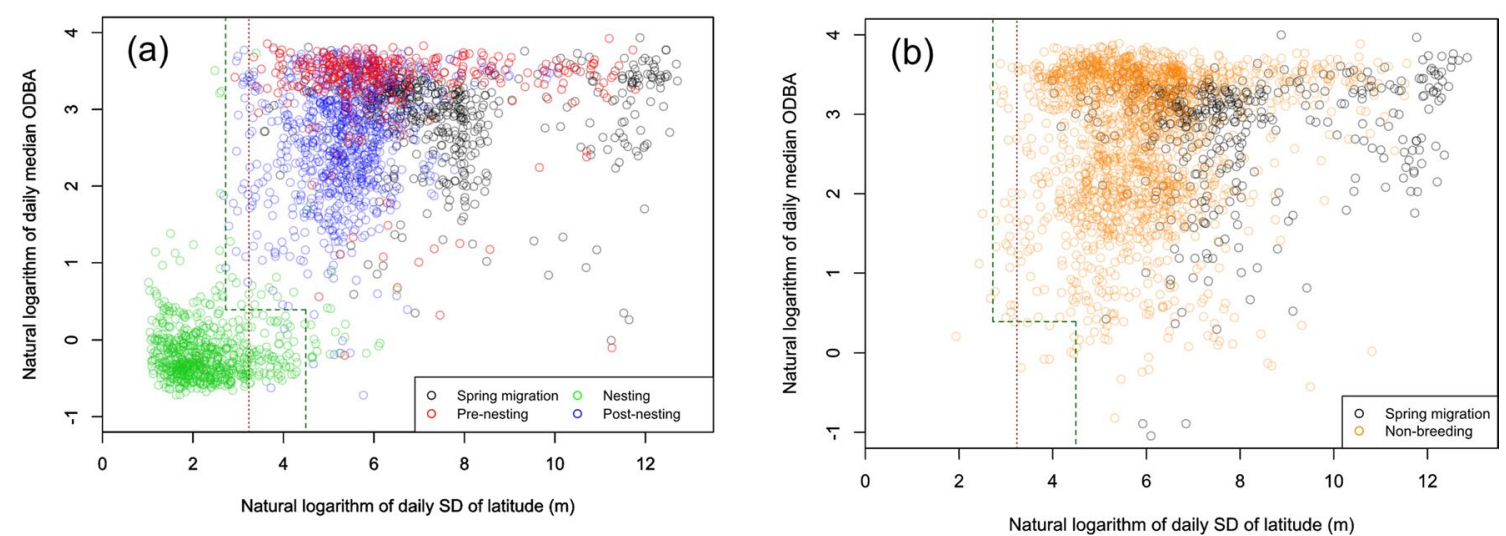

Fig. 4 Body motion and geographic mobility of pink-footed geese during different annual cycle stages (May-July). Per goose, the daily median of the overall dynamic body acceleration (ODBA) was plotted against the daily standard deviation of latitude, both transformed by taking the natural logarithm. Nesting was characterised by days with low body motion and low geographic mobility. Exact cut-off values for the classification of nesting days were determined with Recursive Partitioning and Regression Trees, based on GPS data only (red dotted line) or GPS and accelerometer data combined (green dashed line). a Depicts females that nested ( $n=29$ tracks), while $\mathbf{b}$ depict non-breeding females $(n=25$ tracks). The nesting period was defined based on nest attendance patterns (see Fig. 5). For the other periods, see text 

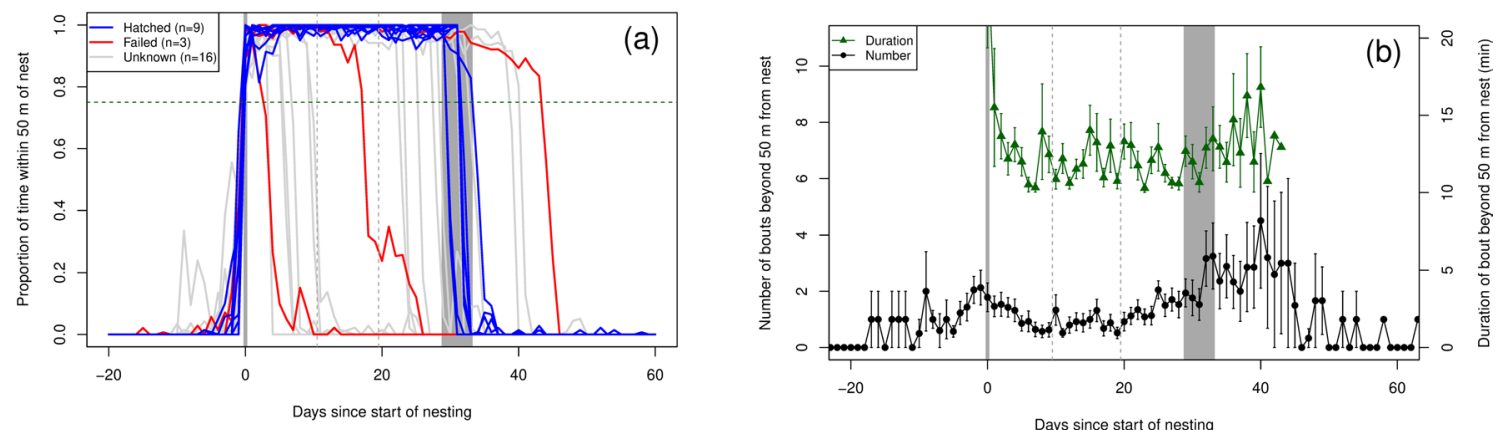

Fig. 5 Nest site attendance and recesses throughout the nesting cycle. Nesting duration, i.e. number of subsequent days with > 75\% (dashed horizontal green line) of time spent within 50 m of the nest, varied between 3 and 44 days for all nests, but between 30 and 34 for successful nests (a). Most nest attempts stopped in the first or last 10 days of the incubation cycle (delineated by dashed vertical grey lines). In these periods, geese went more often beyond $50 \mathrm{~m}$ from the nest, than during the period in between, while the duration of each of those bouts did not vary significantly (b). Grey shading indicates the start and end of successful nesting. In $\mathbf{b}$, means are depicted $\pm \mathrm{SE}$

Table 1 Body motion and geographic mobility of pink-footed geese during different annual cycle stages (May-July)

\begin{tabular}{llll}
\hline Annual cycle stage & Female geese included & Daily median ODBA & Daily SD of latitude (km) \\
\hline Spring migration (from 1 May) & Breeders $(n=29)$ and non-breeders $(n=25)$ & $20.38 \pm 9.23(0.35-54.59)$ & $26.3 \pm 59.0(0.0356-381)$ \\
Pre-nesting & Breeders $(n=29)$ & $30.01 \pm 9.73(0.70-50.57)$ & $6.12 \pm 16.7(0.00857-130)$ \\
Nesting & Breeders $(n=29)$ & $1.08 \pm 2.40(0.49-41.73)$ & $0.0189 \pm 0.0434(0.00278-0.668)$ \\
Post-nesting (until 31 July) & Breeders $(n=29)$ & $17.73 \pm 10.80(0.54-44.41)$ & $0.568 \pm 2.67(0.0145-56.8)$ \\
& Successful, with chicks $(n=9)$ & $15.50 \pm 7.24(1.58-33.95)$ & $0.301 \pm 0.223(0.0156-1.89)$ \\
& Failed, without chicks $(n=16)$ & $19.31 \pm 12.02(0.54-44.41)$ & $0.523 \pm 1.78(0.0145-21.2)$ \\
& Successful, but lost chicks in summer/autumn & $22.51 \pm 11.19(0.49-35.53)$ & $3.09 \pm 6.41(0.116-23.3)$ \\
& $(n=1)$ & & \\
& With unknown hatching success, with & $13.94 \pm 9.65(2.95-39.31)$ & $0.323 \pm 0.401(0.0189-2.56)$ \\
& $30-34$ days nesting $(n=3)$ & & \\
Non-breeding summer (until 31 July) & Non-breeders $(n=25)$ & $20.44 \pm 12.82(0.44-49.01)$ & $1.67 \pm 7.12(0.00690-104)$
\end{tabular}

For five different annual cycle stages, the daily median overall dynamic body acceleration (ODBA) and daily SD of latitude are summarised here as mean \pm SD (range). Nesting was characterised during spring-summer by days with low body motion and low geographic mobility. Post-nesting, breeders with chicks throughout summer showed lower body motion and geographic mobility than breeders without chicks. The nesting period was defined based on nest attendance patterns (see Fig. 1). For the other periods, see text

least 3 consecutive nesting days), and 28 by GPS + ACC. However, from the 25 non-breeding tracks determined by the first approach, four were classified as breeding by GPS-only, while GPS + ACC classified them all correctly as non-breeding. This resulted in a total classification success of breeding/non-breeding status for GPS-only of $87.0 \%$, and for GPS + ACC of $98.1 \%$. The difference was mainly caused by GPS-only missing short attempts and misjudging some non-breeding tracks. Nevertheless, nesting locations estimated by taking the median coordinates of classified nesting days were similar based on GPS-only or GPS + ACC, and approached the groundtruthed nesting locations within few metres (GPS-only: average $2.5 \mathrm{~m}$, SD $0.8 \mathrm{~m}$, range $1.6-3.6 \mathrm{~m}, n=5$ nests, as 1 ground-truthed short nesting attempt was not detected; GPS + ACC: average $2.2 \mathrm{~m}$, SD $1.0 \mathrm{~m}$, range $0.8-3.6 \mathrm{~m}, n=6$ nests).

\section{Deriving nesting success}

The nesting durations of all nesting attempts identified with the first approach varied between 3 and 44 days $(n=$ 28; Fig. 5a). Of these, nests that were confirmed to have hatched showed a nesting duration of 30-34 days $(n=$ 9), while nests that were confirmed to have failed showed nesting durations of 3,18 and 44 days, respectively. The nesting duration of all geese nesting for shorter than 30 days (including those with unknown success) showed a bimodal distribution with most nest attempts stopping after 3-10 days $(n=7)$ or 23-29 days $(n=5)$ and only one after 18 days. Further nesting attempts of unknown success stopped after $32(2 \times), 33,39$ and 41 days. 
During the post-nesting stage, successful breeders with chicks had a lower daily body motion level (LMM, $t=-2.301, d f=23.5, p=0.031$; Table 1 ) and a lower geographic mobility (LMM, for latitude: $t=-2.641$, $d f=20.4, p=0.015$; Table 1 ) than failed breeders without chicks. The body motion and geographic mobility of breeders with unknown nesting success but a nesting duration of 30-34 days, seemed more similar to that of breeders with chicks than failed breeders without chicks (Table 1), but was not significantly different from either group (with chicks: LMM, body motion, $t=-0.279$, $d f=9.7, p=0.79$; LMM, for latitude, $t=0.932, d f=$ 1020, $p=0.35$; without chicks: LMM, body motion, $t=$ $-1.432, d f=17.5, p=0.17$; LMM, for latitude, $t=$ $-1.076, d f=15.6, p=0.30)$.

\section{Variation in nest site attendance}

In the middle of the nesting cycle (day 11-19), geese made $0.87 \pm$ SE 0.18 nest site recesses beyond $50 \mathrm{~m}$ from the nest per day, which tended to be lower, but not significantly, than the $1.1 \pm \mathrm{SE} 0.14$ recesses per day in the first period (day $0-10, d f=719, t=1.882, p=$ 0.060 ) and was significantly lower than the $1.6 \pm$ SE 0.15 recesses per day in the last period (day 20-29, $d f=709$, $t=4.537, p<0.001$; Fig. 5b). The duration of these bouts was not different between the middle period (18.5 $\pm \mathrm{SE}$ $4.7 \mathrm{~min})$ and the first period (19.5 $\pm \mathrm{SE} 0.73 \mathrm{~min}, d f=$ $400, t=1.342, p=0.18)$ or the last period (18.7 $\pm \mathrm{SE}$ $0.76 \min , d f=399, t=0.222, p=0.82$; Fig. $5 \mathrm{~b}$ ).

\section{Discussion}

We have presented a method to use GPS-tracking and accelerometry to remotely identify pink-footed goose nesting attempts and their locations, the start and end of each nesting attempt, and thus also an indication of nesting success given by the duration of nest site attendance. We also showed that $>98 \%$ of nesting days could be classified correctly by two main movement characteristics of the breeding phase of the annual cycle (i.e. low geographic mobility and low body motion) and that including accelerometry especially helps to detect short nesting attempts correctly.

Our main approach acquires a high level of precision and can detect nest attempts as short as 3 days, which is not easily achievable with methods based on only GPS without additional sensor data. For example, [21] suggest for three bird species that nesting attempts below 7-14 days can usually not be detected based on GPStracking data, and [19] and [20] use for two goose species a minimum duration of 10 days. The power of including sensor data in the analysis lies in the fact that one can specifically select GPS-fixes (or days) with a certain body motion of interest and exclude those that are part of other activities than nesting, e.g., foraging or preening. This was apparent in our second approach, where accelerometry increased the likelihood that both short breeding attempts and non-breeding tracks are correctly classified, whereas the goose's spatial behaviour per se could be ambiguous (i.e. low geographic mobility for a short time). This is useful when one aims to study the timing of breeding, or to distinguish a low breeding propensity from a high breeding failure. We argue that including accelerometer data, if available, can increase the power of nest detection.

By evaluating the proportion of time spent at such locations, our method could also distinguish roosting areas from nesting attempts that lasted at least three days. A goose may roost for longer periods, but apparently rarely on exactly the same spot during subsequent days. Roosting behaviour may differ for other species, e.g., raptors, which may have specific roosting trees [47] or gulls, which consistently roost on the same breakwater [48]. For such species, the body orientation as measured by an accelerometer would still be useful to detect nesting sites, e.g., straight up during roosting, versus horizontal during nesting in raptors [49]. Our study provides a reliable method that enables the study of avian breeding in remote environments, which is often logistically challenging with classic observations.

The nesting period is unique in its virtual absence of movement. Although this would suggest low energy expenditure [42], nesting is energetically a demanding time for geese, resulting in large body mass loss as egg production is costly and foraging opportunities are traded off against incubation activity and nest defence [50]. The higher daily activity levels we found in the prenesting stage may reflect active foraging for large parts of the day [27], whereas movement patterns in the postnesting stage depended on family status. Geese with chicks had lower body motion and lower geographic mobility than geese without chicks, probably the result of regularly brooding the chicks in the field and flying less. The large geographic mobility that distinguishes spring migration results both from daily movements between roost sites and foraging sites within a stop-over, as well as directional movements between stop-overs [25].

The nesting duration of successful nests concentrated, as expected, at 30-34 days, whereas unsuccessful nests showed both shorter and longer nesting durations. For further studies, one may assume that any goose with unknown nesting success but a nesting duration of 30-34 days is successful, as post-nesting body motion and geographic mobility of such geese seemed more similar to those with hatched nests than failed nests. Nesting durations shorter than 30 days are likely caused by nest predation or abandonment [1]. Also 
human disturbance might indirectly lead to nest loss by predation [51]. Nest losses before normal hatching time occurred in our study predominantly in the first and last ten days of a normal nesting cycle. This pattern may be explained by the slightly higher recorded frequency of nest site recesses in these periods, compared to the period in between. Nest recesses, to forage or drink, expose the nest to predators [3]. Because our GPS-fixes include GPS error, we cannot report exactly how far a goose was situated from its nest during a bout. Also, recesses shorter than $10 \mathrm{~min}$ can be missed due to the logging interval. Still, our analysis is expected to give a reliable qualitative view on (far and long) nest site recesses. Moreover, the same pattern of nest attendance over the incubation cycle was reported based on direct observations and showed that females spend more time foraging in the first week and last days of nesting than in the period in between [28]. In contrast, dark-bellied Brent geese (Branta bernicla bernicla) and greater snow geese (Chen caerulescens atlantica) were found to make fewer and shorter recesses in the last days before hatching $[50,52]$. Differences in incubation constancy between species may result from the interplay of remaining body stores (thus, the need for foraging trips), foraging opportunities close to the nest, and the local risk of nest predation (thus, the need to stay on the nest). Future studies using our method could evaluate how nest attendance and nesting duration vary with environmental factors and may change with arctic warming.

Nesting durations longer than 34 days are longer than expected, even for large clutches and may indicate that the goose was incubating unhatchable eggs. Pink-footed geese may continue to incubate an unhatched egg for 3 more days after all other eggs have already hatched, before they leave with the brood [28]. However, when the whole clutch is unhatchable, many bird species extend their incubation duration by at least 50\% [53]. A nest with solely unhatchable eggs is expected to be rare, but can, for example result from predation of already-hatched chicks-in our study one nest was incubated for 44 days, but contained only one egg on day 43. Egg hatching failure can be caused by environmental pollutants resulting in embryonal death or deformities [e.g., 54, 55]. To detect such phenomena, the study of individual breeding attempts by GPS-tracking and accelerometry, as facilitated by this study, is a valuable addition to the extensive monitoring of productivity on the population level [36]. Our study also highlights the importance of characterising successful nesting attempts not solely by a minimum nesting period, but by a defined range in nesting periods.

Technological advances in tracking devices make that GPS data are increasingly accompanied by sensor data.
Combining GPS with accelerometer data can increase the power to detect short nesting attempts and determine the timing, location and success of breeding. Studying avian breeding in the changing remote arctic environment is of great interest and will be subject of future research, facilitated by the method presented in this paper.

\section{Conclusions}

Nesting attempts of Arctic-breeding pink-footed geese (as short as 3 days) and their locations can be derived remotely and accurately from GPS-tracking and accelerometry, while nesting success is indicated by the nesting duration. We predict that this method can be generalised to other (precocial) bird species with similar incubation behaviour.

\section{Abbreviations}

ACC: Accelerometry; ODBA: Overall dynamic body acceleration; VeDBA: Vectorial dynamic body acceleration; SD: Standard deviation.

\section{Acknowledgements \\ We are grateful to people who helped catch geese in Svalbard (Christian Sonne, Peter de Vries, Koen Nolet, Ove Martin Gundersen, John Frikke, Cornelia Jaspers, Kevin Kuhlmann Clausen, Cecilia Sandström, Dies Snoodijk and the crew of RV "Ulla Rinman") and in Finland (Jorma Pessa, Tuomas Väyrynen, Esko Pasanen, Aija Lehikoinen, Christanne de Vries, Jorma Siira, Matti Tolvanen, Antti Piironen, Christian Sonne, Lars Haugaard, Jens Peter Hounisen, Michael Schmidt, Niels-Erik Jørgensen, Maël Charbonneaux, Kalle Hiekkanen). Further we thank observers, especially Aija Lehikoinen, Jørgen Peter Kjeldsen, Ole Amstrup, Fred Cottaar, Eckhart Kuijken, Christine Verscheure, Leo Schilperoord and Mirjam Schilperoord-Huisman, Ove M. Gundersen, Kevin K. Clausen, Kent Larsson and Lennart Eriksson, for observing tagged geese in the field, and Martijn van der Sluijs and Christa Mateman for the molecular sexing. Fieldwork in Norway was kindly facilitated by Skogn Folkehøgskole, Skogn. We thank Stijn Schreven for editing figure 1, and two anonymous referees for their constructive comments.}

\section{Authors' contributions}

Conceptualisation, KS, BAN; F, KS, CS, JM, BAN; formal analysis, KS; writingoriginal draft KS; writing — review and editing, KS, CS, JM, BAN; supervision, JM., BAN. All authors read and approved the final manuscript.

\section{Funding}

Our work was supported by grants from the Netherlands Polar Programme of the Dutch Research Council to BAN (ALWPP.2016.024), from the Svalbard Environmental Protection Fund to JM (No. 17/88) and from the Academy Ecology Fund of the Royal Netherlands Academy of Arts and Sciences to KHTS. The funding body had no role in the design of the study and collection, analysis, and interpretation of data or in writing the manuscript.

\section{Availability of data and materials}

The datasets generated and/or analysed during the current study are not publicly available yet, pending publication of another article, but are available from the corresponding author on reasonable request.

\section{Declarations}

\section{Ethics approval and consent to participate}

Permits to catch and tag geese in Svalbard were granted by the Norwegian Food Safety authority (Mattilsynet) (to Aarhus University, 17/210528), by the Governor of Svalbard (17/01420-4) and Longyearbyen Lokalstyre (2018/3475-X70), in Finland by Etelä-Suomen aluehallintovirasto (to Aarhus University, 
ESAVI/1924/2018 and ESAVI/1880/2018) and Varsinais-Suomen elinkeino-, liikenne- ja ympäritsökeskus (to Jorma Pessa, VARELY/551/2018).

\section{Consent for publication}

Not applicable.

\section{Competing interests}

The authors declare that they have no competing interests.

\section{Author details}

'Department of Animal Ecology, Netherlands Institute of Ecology (NIOOKNAW), Droevendaalsesteeg 10, 6708 PB Wageningen, The Netherlands. ${ }^{2}$ Norwegian Institute for Nature Research (NINA), 7485 Trondheim, Norway. ${ }^{3}$ Department of Biology, Norwegian University of Science and Technology (NTNU), 7491 Trondheim, Norway. ${ }^{4}$ Department of Bioscience, Aarhus University, 8410 Rønde, Denmark. ${ }^{5}$ Department of Theoretical and Computational Ecology, Institute for Biodiversity and Ecosystem Dynamics, University of Amsterdam, Science Park 904, 1098 XH Amsterdam, The Netherlands.

Received: 2 February 2021 Accepted: 21 June 2021

Published online: 13 July 2021

\section{References}

1. Anderson HB, Madsen J, Fuglei E, Jensen GH, Woodin SJ, van der Wal $R$. The dilemma of where to nest: influence of spring snow cover, food proximity and predator abundance on reproductive success of an arcticbreeding migratory herbivore is dependent on nesting habitat choice. Polar Biol. 2014;38:153-62.

2. Perrins CM. The timing of birds' breeding seasons. Ibis. 1970;112:242-55

3. Madsen J, Tamstorf M, Klaassen M, Eide N, Glahder C, Rigét F, Nyegaard $\mathrm{H}$, Cottaar F. Effects of snow cover on timing and success of reproduction in high-Arctic Pink-footed Geese Anser brachyrhynchus. Polar Biol. 2007;30:1363-72. https://doi.org/10.1007/s00300-007-0296-9.

4. Ross MV, Alisauskas RT, Douglas DC, Kellett DK. Decadal declines in avian herbivore reproduction: density-dependent nutrition and phenological mismatch in the Arctic. Ecology. 2017;98:1869-83.

5. Brook RW, Leafloor JO, Abraham KF, Douglas DC. Density dependence and phenological mismatch: consequences for growth and survival of sub-arctic nesting Canada Geese. Avian Conserv Ecol. 2015;10:1.

6. Lameris TK, van der Jeugd HP, Eichhorn G, Dokter AM, Bouten W, Boom MP, Litvin KE, Ens BJ, Nolet BA. Arctic geese tune migration to a warming climate but still suffer from a phenological mismatch. Curr Biol. 2018;28:2467-73.

7. Ross MV, Alisauskas RT, Douglas DC, Kellett DK, Drake KL. Densitydependent and phenological mismatch effects on growth and survival in lesser snow and Ross's goslings. J Avian Biol. 2018;49:e01748.

8. Reed TE, Grøtan V, Jenouvrier S, Sæther BE, Visser ME. Population growth in a wild bird is buffered against phenological mismatch. Science. 2013;340(6131):488-91.

9. Nolet BA, Schreven KHT, Boom MP, Lameris TK. Contrasting effects of the onset of spring on reproductive success of Arctic-nesting geese. Auk. 2020;137(1):ukz063.

10. Lack D. Ecological adaptations for breeding in birds. London: Methuen; 1968.

11. Visser ME, van Noordwijk AJ, Tinbergen JM, Lessells CM. Warmer springs lead to mis-timed reproduction in Great Tits (Parus major). Proc R Soc Lond B. 1998;265:1867-70.

12. Doiron M, Gauthier G, Levesque E. Trophic mismatch and its effects on the growth of young in an Arctic herbivore. Glob Change Biol. 2015;21(12):4364-76. https://doi.org/10.1111/gcb.13057.

13. Høye TT, Post E, Meltofte H, Schmidt NM, Forchhammer MC. Rapid advancement of spring in the High Arctic. Curr Biol. 2007;17(12):R449-51.

14. Cohen J, Screen JA, Furtado JC, Barlow M, Whittleston D, Coumou D, Francis J, Dethloff K, Entekhabi D, Overland J, Jones J. Recent Arctic amplification and extreme mid-latitude weather. Nat Geosci. 2014;7(9):627.

15. Alzate Valejo A. From local adaptation to range sizes: ecological and evolutionary consequences of dispersal. PhD thesis, Groningen, The Netherlands: University of Groningen; 2018.
16. Davidson SC, Bohrer G, Gurarie E, LaPoint S, Mahoney PJ, Boelman NT, et al. Ecological insights from three decades of animal movement tracking across a changing Arctic. Science. 2020;370(6517):712-5.

17. Dokter AM, Fokkema W, Bekker SK, Bouten W, Ebbinge BS, Müskens G, Olff $\mathrm{H}$, van der Jeugd HP, Nolet BA. Body stores persist as fitness correlate in a long-distance migrant released from food constraints. Behav Ecol. 2018;29(5):1157-66. https://doi.org/10.1093/beheco/ary080.

18. Nuijten RJM, Gerrits T, Shamoun-Baranes J, Nolet BA. Less is more: onboard lossy compression of accelerometer data increases biologging capacity. J Anim Ecol. 2020;89(1):237-47. https://doi.org/10.1111/13652656.13164.

19. de Boer R, Bauer S, van der Jeugd HP, Ens BJ, Griffin L, Cabot D, Exo KM, Nolet BA, Kölzsch A. A comparison of spring migration between three populations of Barnacle Geese Branta leucopsis using GPS satellite transmitters. Limosa. 2014;87:99-106.

20. Kölzsch A, Müskens GJDM, Szinai P, Moonen S, Glazov P, Kruckenberg H, Wikelski M, Nolet BA. Flyway connectivity and exchange primarily driven by moult migration in geese. Mov Ecol. 2019;7(1):1-11.

21. Picardi S, Smith BJ, Boone ME, Frederick PC, Cecere JG, Rubolini D, Serra L, Pirrello S, Borkhataria RR, Basille M. Analysis of movement recursions to detect reproductive events and estimate their fate in central place foragers. Mov Ecol. 2020;8(1):1-14.

22. Wilson RP, White CR, Quintana F, Halsey LG, Liebsch N, Martin GR, Butler PJ. Moving towards acceleration for estimates of activity-specific metabolic rate in free-living animals: the case of the cormorant. J Anim Ecol. 2006;75(5):1081-90.

23. Scott DA, Rose PM. Atlas of Anatidae Populations in Africa and Western Eurasia. Wageningen, The Netherlands: Wetlands International; 1996.

24. Cramp S, Simmons KEL. Handbook of the birds of Europe, the Middle East, and North Africa: the birds of the Western Palearctic, vol. I. Oxford: Oxford University Press; 1977.

25. Chudzińska ME, Nabe-Nielsen J, Nolet BA, Madsen J. Foraging behaviour and fuel accumulation of capital breeders during spring migration as derived from a combination of satellite- and ground-based observations. J Avian Biol. 2016:47:563-74.

26. Glahder CM, Fox AD, Hübner CE, Madsen J, Tombre IM. Pre-nesting site use of satellite transmitter tagged Svalbard Pink-footed Geese Anser brachyrhynchus. Ardea. 2006;94(3):679-90.

27. Fox AD, Francis IS, Bergersen E. Diet and habitat use of Svalbard Pinkfooted Geese Anser brachyrhynchus during arrival and pre-breeding periods in Adventdalen. Ardea. 2006;94(3):691-9.

28. Inglis IR. The breeding behaviour of the Pink-footed Goose: behavioural correlates of nesting success. Anim Behav. 1977;25:747-64.

29. Bauer KM, von GlutzBlotzheim UN. Handbuch der Vögel Mitteleuropas, vol. 2. Frankfurt: Akademische Verlagsgesellschaft; 1968.

30. Bezzel E. Kompendium der Vögel Mitteleuropas: NonpasseriformesNichtsingvögel. Wiesbaden: Aula-verlag; 1985.

31. Witherby HF, Jourdain FCR, Ticehurst NF, Tucker BW. The handbook of British Birds. Watford Edinburgh: JJ Gray and Sun Printers Ltd; 1944.

32. Del Hoyo J, Elliott A, Sargatal J. Handbook of the Birds of the World, vol. 1. Barcelona: Lynx Edicions; 1992.

33. Lazarus J, Inglis IR. The breeding behaviour of the Pink-footed Goose: parental care and vigilant behaviour during the fledging period. Behaviour. 1978;65(1/2):62-88.

34. Clausen KK, Schreven KHT, Madsen J. Effects of capture and marking on behaviour of moulting Pink-footed Geese Anser brachyrhynchus on Svalbard. Wildfowl. 2020;70:13-29.

35. Jensen $\mathrm{GH}$, Tombre IM, Madsen J. Environmental factors affecting numbers of Pink-footed Geese Anser brachyrhynchus utilising an autumn stopover site. Wildl Biol. 2016;22(5):183-93.

36. Madsen J, Jensen GH, Cottaar F, Amstrup O, Bak M, Bakken J, Balsby TTJ, Kjær Christensen T, Clausen KK, Frikke J, Gundersen OM, Kjeldsen JP, Koffijberg K, Kuijken E, Månsson J, Nicolaisen PI, Haaning Nielsen H, Nilsson L, Reinsborg T, Ødegaard PI, Pessa J, Shimmings P, Tombre I, Verscheure C. Svalbard Pink-footed Goose Population status report 2017-2018. Bonn, Germany: AEWA EGMP Technical Report; 2018.

37. Fridolfsson AK, Ellegren $\mathrm{H}$. A simple and universal method for molecular sexing of non-ratite birds. J Avian Biol. 1999;30:116-21.

38. Griffiths R, Double MC, Orr K, Dawson RJ. A DNA test to sex most birds. Mol Ecol. 1998;7(8):1071-5. 
39. Ebbinge BS, Buij R, de Vries L, Moonen S, van Randen Y, Müskens G, van der Jeugd H, Koffijberg K, Voslamber B, Roosenschoon O, Kramer J. The website geese.org, an interactive database to report marked waterfowl. Goose Bulletin. 2020;25:11-8.

40. Gupte PR, Koffijberg K, Müskens GJDM, Wikelski M, Kölzsch A. Family size dynamics in wintering geese. J Ornithol. 2019;160:363-75.

41. Klaassen $M$, Hahn $\mathrm{S}$, Korthals $\mathrm{H}$, Madsen J. Eggs brought in from afar: Svalbard-breeding Pink-footed Geese can fly their eggs across the Barents Sea. J Avian Biol. 2017:48:173-9. https://doi.org/10.1111/jav.01364.

42. Wilson RP, Börger L, Holton MD, Scantlebury DM, Gómez-Laich A, Quintana F, Rosell F, Graf PM, Williams H, Gunner R, Hopkins L, Marks N, Geraldi NR, Duarte CM, Scott R, Strano MS, Robotka H, Eizaguirre C, Fahlman A, Shepard ELC. Estimates for energy expenditure in free-living animals using acceleration proxies: a reappraisal. J Anim Ecol. 2020;89(1):161-72.

43. Bates D, Mächler M, Bolker BM, Walker SC. Fitting linear mixed-effects models using Ime4. J Stat Softw. 2015;67(1):1-48. https://doi.org/10 18637/jss.v067.i01.

44. Kuznetsova A, Brockhoff PB, Christensen RHB. ImerTest package: tests in linear mixed effects models. J Stat Softw. 2017;82(13):1-26. https://doi. org/10.18637/jss.v082.i13.

45. Therneau T, Atkinson B, Ripley B. Recursive Partitioning and Regression Trees. 2019. https://cran.r-project.org/package=rpart. Accessed 15 May 2021.

46. R Core Team. R: A language and environment for statistical computing. R Foundation for Statistical Computing, Vienna, Austria. 2020. https:// www.R-project.org/. Accessed 01 Jan 2020.

47. Deng WH, Wei G, Guang-Mei Z. Nest and roost habitat characteristics of the Grey-faced Buzzard in northeastern China. J Raptor Res. 2003:37(3):228-35.
48. van Donk SC. A gull's portrait: On individual foraging strategies and their fitness consequences. PhD Thesis, The Netherlands: Vrije Universiteit Amsterdam; 2020.

49. Newton I. The sparrowhawk. Calton: A\&C Black; 1986.

50. Spaans B, van't Hoff CA, van der Veer W, Ebbinge BS. The significance of female body stores for egg laying and incubation in Dark-bellied Brent Geese Branta bernicla. Ardea. 2007;95(1):3-15.

51. Madsen J, Tombre I, Eide NE. Effects of disturbance on geese in Svalbard: implications for regulating increasing tourism. Polar Res. 2009;28(3):376-89.

52. Poussart C, Gauthier G, Larochelle J. Incubation behaviour of greater snow geese in relation to weather conditions. Can J Zool. 2001;79(4):671-8

53. Skutch AF. The constancy of incubation. Wilson Bull. 1962;74(2):115-52.

54. Skorupa JP, Ohlendorf HM. Contaminants in drainage water and avian risk thresholds. In: Dinar A, Zilberman D, editors. The economics and management of water and drainage in agriculture. New York: Springer; 1991.

55. van Oosten $\mathrm{HH}$, van den Burg AB. Dioxines: een niet-onderkend probleem voor zangvogels? The Netherlands: Report Stichting Bargerveen; 2014

\section{Publisher's Note}

Springer Nature remains neutral with regard to jurisdictional claims in published maps and institutional affiliations.
Ready to submit your research? Choose BMC and benefit from:

- fast, convenient online submission

- thorough peer review by experienced researchers in your field

- rapid publication on acceptance

- support for research data, including large and complex data types

- gold Open Access which fosters wider collaboration and increased citations

- maximum visibility for your research: over $100 \mathrm{M}$ website views per year

At $\mathrm{BMC}$, research is always in progress.

Learn more biomedcentral.com/submissions 